\title{
Emociones cromáticas: análisis de la percepción de color basado en emociones y su relación con el consumo de moda*
}

\author{
Sonia M. Peláez Becerra** \\ Paula Gómez Gómez z*^ \\ Miguel A. Becerra***
}

Recibido: 2015 08-10 Enviado a pares: 2015-08-22

Aprobado por pares: 2015-09-22 Aceptado: 2015-10-01

\section{Resumen}

La globalización del mercado actual exige que los productos sean novedosos, comerciales, funcionales y altamente llamativos, para despertar así en el consumidor la atención suficiente y lograr que se dé el momento de la compra, con la previa identificación de su necesidad y la aceptación del producto. Caracterizar al consumidor es complejo, y deben ser analizados diferentes aspectos como el color y las emociones. En este trabajo se estableció, la relación que existe entre la emoción, el color y el objeto moda, identificando las variables que motivan al consumidor en el momento de su compra. Para ello se aplicó una encuesta sobre una población universitaria en Colombia y, adicionalmente, mediante la base de datos DEAP perteneciente a 4 universidades de Europa (Queen Mary University de Londres, University of Twente de Holanda, University of Geneva y al EPFL de Suiza), que relaciona emociones y vídeos en una población universitaria de Europa, se hizo un análisis del color en la ambientación de estos y se demostró la influencia del color utilizando el gráfico de Geneve. Los resultados obtenidos demostraron una fuerte influencia del color en la actitud de consumo y del color sobre las emociones, aunque no se logró concluir que las emociones afecten el momento de adquisición por parte del consumidor.

Palabras clave: color, consumidor, emoción, moda, percepción.

* $\quad$ El proyecto se encuentra asociado al proyecto de investigación titulado "Análisis de la percepción del color sobre las emociones por medio de técnicas inteligentes a partir de señales EEG", del Centro de Investigación de la Institución Universitaria Salazar y Herrera, Medellín-Colombia.

** Maestría en Diseño de Producto con Textil Tecnológico. Diseñadora gráfica, Universidad Pontificia Bolivariana, docente de la Escuela de Artes de la Institución Universitaria Salazar y Herrera, coordinadora del Semillero de Investigación Infográfos, miembro del grupo Código de la Escuela de Artes. sonia.pelaez@iush.edu.co

*** Comunicadora gráfica publicitaria, Universidad de Medellín; magíster en Dirección de Marketing. Universidad de Viña del Mar. Docente de la Institución Universitaria Salazar y Herrera - p.gomez@iush.edu.co

**** Ingeniero electrónico, Universidad Nacional de Colombia. Especialista en Pedagogía virtual, UCN. Maestría en Automatización y Control Industrial, ITM. Doctorado en Ingeniería (en curso), Universidad Nacional de Colombia; docente Escuela de Ingeniería, en los programas Ingeniería Electrónica e Ingeniería de Sistemas, líder del grupo de investigación GEA, par académico nacional e internacional e investigador asociado de Colciencias. miguel.becerra@ iush.edu.co 


\title{
Chromatic Emotions: Analysis of color perception based on emotions and its relationship with consumption of fashion
}

\begin{abstract}
The globalization of the marketplace, requires that products are innovative, commercial, functional and highly striking, to wake up as well in the consumer, the sufficient attention and achieve that you give the time of the purchase, with prior identification of your need and the acceptance of the product. Characterize the consumer is complex and different aspects like color and emotions must be analyzed. In this work is established, the relationship between emotion, color and fashion object, identifying variables that motivate the consumer at the time of your purchase. So a survey was applied on a university population in Colombia and Additionally, through database belonging DEAP 4 universities in Europe (Queen Mary University of London, University of Twente, Netherlands, University of Geneva and at the EPFL in Switzerland), that related emotions and videos in a university population of Europe, made an analysis of the color in the background of these and showed the influence of the color using the graph of Genève. The results obtained showed, a strong influence of the color in the attitude of consumption and the color on the emotions, although it was not possible to conclude that emotions affect the time of purchase by the consumer.
\end{abstract}

Key words: Color, Consumer, Emotion, Fashion, Perception. 


\section{Introducción}

El proceso de aceptar o rechazar un producto es de naturaleza multidimensional y su estructura es dinámica, pues se da entre miembros en el interior de un grupo e individualmente, en diferentes contextos y períodos de tiempo. Dentro de estas variables está el color y sus características como la iluminación, el brillo o intensidad, y la temperatura, que afectan no solo las emociones, sino que producen también oscilaciones cerebrales, que se manifiestan en el comportamiento del individuo (Shin, y otros, 2015), e influyen en la aceptación de los objetos (Álvarez Lara, 2011) y afectan las intenciones de compra por parte del consumidor, por lo que se hace necesario identificar cuál es el alcance de la influencia del color sobre las emociones que incitan al acto de consumo, provocando reacciones diferentes, las cuales están mediadas por las asociaciones cognitivas y afectivas del consumidor (Babin, Hardesty, E Suter, 2003).

En Satake (2011) se establece el color como un relevante factor de influencia en el consumo y con alta correlación con las emociones, por lo que resulta importante el análisis de la percepción del color y su relación con el objeto de moda, basado en las emociones, teniendo en cuenta diferentes aspectos como la percepción visual o atención, la cual permite la clasificación de la intensidad de cada señal, por medio de procesos mentales, a partir del análisis, la comparación, la evaluación y comprensión de la información y su significado (Mangun, 2013). Este proceso de integración de las probabilidades, el procesamiento y posterior conocimiento basado en el recuerdo y las experiencias almacenadas es denominado de diferentes formas como: (i) Psicología de la Gestalt (Desolneux, Moisan, \& Morel, 2008), donde Gestalt significa cierre o completación de un ciclo, proceso que es conocido como pregnancia o ley de lo compacto (Prägnaz) (Moszkowicz, 2011); (ii) proceso Insight (Dr. Simon $\&$ Goes, 2013), y (iii) cierre del campo psicológico para elaborar una estructura (Oviedo, 2004).

Otros estudios, pero en el ámbito del mercado, resaltan la importancia del análisis de la respuesta emocional de los consumidores ante las tendencias de moda actual, la cual se encuentra relacionada con el color y que genera una carga emocional, que ocasiona que se fijen las experiencias a partir de este en la memoria, a razón de que sus efectos no están regidos por normas y es imposible controlarlos. El color posee gran fuerza expresiva y esto lo convierte en un lenguaje (Puig Borrás, 2011), que se puede leer y así prever, a manera de tendencia para las próximas temporadas, con base en los resultados analizados y las emociones producidas (Cho, 2005). En particular, la tendencia de color de moda es muy volátil, esto a causa de que los individuos emiten juicios estéticos de diferente valor de agradabilidad dependiendo de cada tonalidad y de cómo les afecta (Santalla de Banderalli, 2011). Tan alta es la importancia de este tipo de análisis que recientemente se ha despertado un alto interés por parte de la comunidad científica de las ciencias de la computación para dar una valoración más cuantitativa aplicando diferentes técnicas de inteligencia artificial para poder realizar predicciones (Yu, Hui, \& Choi, 2012); sin embargo, la precisión proporcionada por estos sistemas es aún controversial. 
En este estudio se presenta un análisis comparativo de las emociones y el color a partir de resultados estadísticos obtenidos de encuestas, relaciones de emociones y color, y con muestras de vídeo para determinar la influencia del color en la decisión de compra demostrando la dependencia entre el color, la emoción y el consumo, pero con algunas limitaciones.

El artículo se encuentra distribuido en 6 secciones: en la sección 2 se presentan los principales conceptos y teorías en torno a color, emociones y consumo; en la sección 3 se expone la metodología aplicada y en la sección 4 se discuten los resultados. Finalmente, en la sección 5 de conclusiones, se presentan las principales contribuciones, inconvenientes y posible trabajo futuro.

\section{Materiales y métodos}

\section{Percepción del color}

El individuo constantemente es estimulado por el entorno que lo rodea, y cada objeto emite una señal estimulante al sujeto, la cantidad de información es variable y este la recibe simultáneamente, reaccionando ante cada estímulo de forma particular; a este proceso de recibir información del exterior a través de los órganos del cuerpo se le llama percepción; en el caso de la excitación del órgano de la visión se denomina percepción visual (Gonzales Cuasante E Cuevas Riaño, 2005).

A diferencia de la mayoría de los animales, los seres humanos se basan con mayor fuerza, en el sentido de la visión para percibir el mundo (Morris, 2005).

El sistema de visión humana permite ver una extensa gama de colores, en donde intervienen el matiz, la saturación y el brillo; esto, debido a que la percepción del color, depende principalmente de la longitud de onda de la luz, mientras que la amplitud incide en el brillo y la pureza, en la saturación (Gonzales Cuasante E Cuevas Riaño, 2005).

En la gráfica 1 se presentan dos teorías que dan explicación al fenómeno de la percepción del color: una es la teoría del proceso oponente, que sostiene que los receptores están especializados para responder a cualquier miembro de los tres pares de colores básicos: rojo y verde, amarillo y azul y blanco y negro (luz y oscuridad). La otra es la teoría tricromática de la visión cromática, que sostiene que el ojo contiene tres tipos de receptores de color, que responden a la luz roja, verde o azul. El encéfalo es el que posibilita detectar cualquier color, incluso las sutiles diferencias entre ellos (Gonzales Cuasante \& Cuevas Riaño, 2005).

\section{Teoría de la Gestalt}

La teoría de la Gestalt es definida como la existencia de un factor de cierre, la reorganización o la redefinición del "todo" estructurado, combinando flexibilidad, análisis y síntesis, aun cuando otras condiciones estarían ligadas al grado de complejidad o de enlace de las estructuras conceptuales que el individuo es capaz de realizar (Novaes, 1973) 

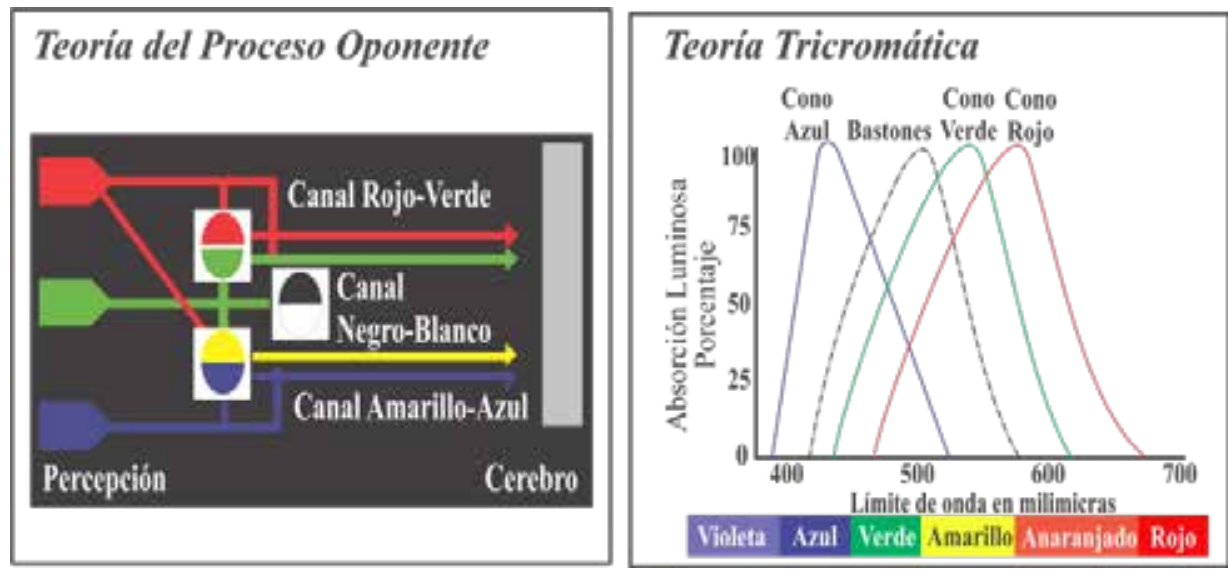

GRÁFICA 1 Diagrama de la teoría de oponentes y Teoría Tricromática.

Fuente: Gonzales Cuasante \& Cuevas Riaño, 2005

La psicología de la Gestalt, plantea que: "Los hechos psíquicos son formas, es decir, unidades que se individualizan y se limitan en el campo espacial y en un tiempo de percepción o de representación". En este caso, para la percepción, las formas son un conjunto de factores determinados de un grupo; algunas de sus propiedades se conservan y no cambian y otras de cierta manera afectan a todos esos factores (Moszkowicz, 2011).

A raíz de algunas investigaciones y experiencias, los psicólogos de la Gestalt han elaborado leyes referentes al campo perceptivo; tales leyes son: 1) Ley de pregnancia o buena forma, 2) Ley de proximidad, 3) Ley de similitud o semejanza, 4) Ley de la dirección, 5) Ley de cierre y 5) Ley de figura y fondo. Dichas leyes son las que permiten darle un orden al proceso perceptual (Köhler, 1972).

\section{Variables de consumo}

Para analizar qué estrategias de marketing y las razones por las cuales se impulsa el acto de consumo de determinados productos, servicios o marcas por parte del consumidor (Roger Blackwell, 2009), se debe identificar, además de las variables de su comportamiento, la existencia de cuatro factores psicológicos que también intervienen en su conducta: como la personalidad y el autoconcepto, la motivación, la percepción y el aprendizaje; estos cuatro pilares de comportamiento hacen parte del eje creativo que ayuda a generar las estrategias de mercadeo que influyen en el acto de compra. Sin embargo, cuando la psicología es aplicada al estudio del comportamiento del consumidor, se pretende descubrir las reacciones de este ante las estrategias de mercadeo, por lo cual se considera cada persona como el resultado de la interacción de una multiplicidad de variables integradas por muchos componentes, lo que supone un mundo infinito de combinaciones. Esta infinidad de combinaciones lleva a que el individuo sea único y diferente. Así, las variables que se involucran en el comportamiento del consumidor se clasifican en tres grupos (Cueva, 2011) (gráfica 2 y 3) así: 
Variables de influencia: son todas las variables a las que los individuos están sujetos como seres inteligentes y, por lo tanto, influyen en su comportamiento.

Variables de procesamiento: son todas aquellas variables que se dan en el pensamiento del individuo como las sensaciones, percepciones del color, del sabor y de olores, entre otros, motivaciones y actitudes, que también influyen en la personalidad o el estilo de vida.

Variables de resultado: son aquellas variables que determinan el comportamiento de la compra como la retención de la publicidad (comportamiento muy influenciado por el color), la lealtad de marca, el estatus, etc. (Cueva, 2011).

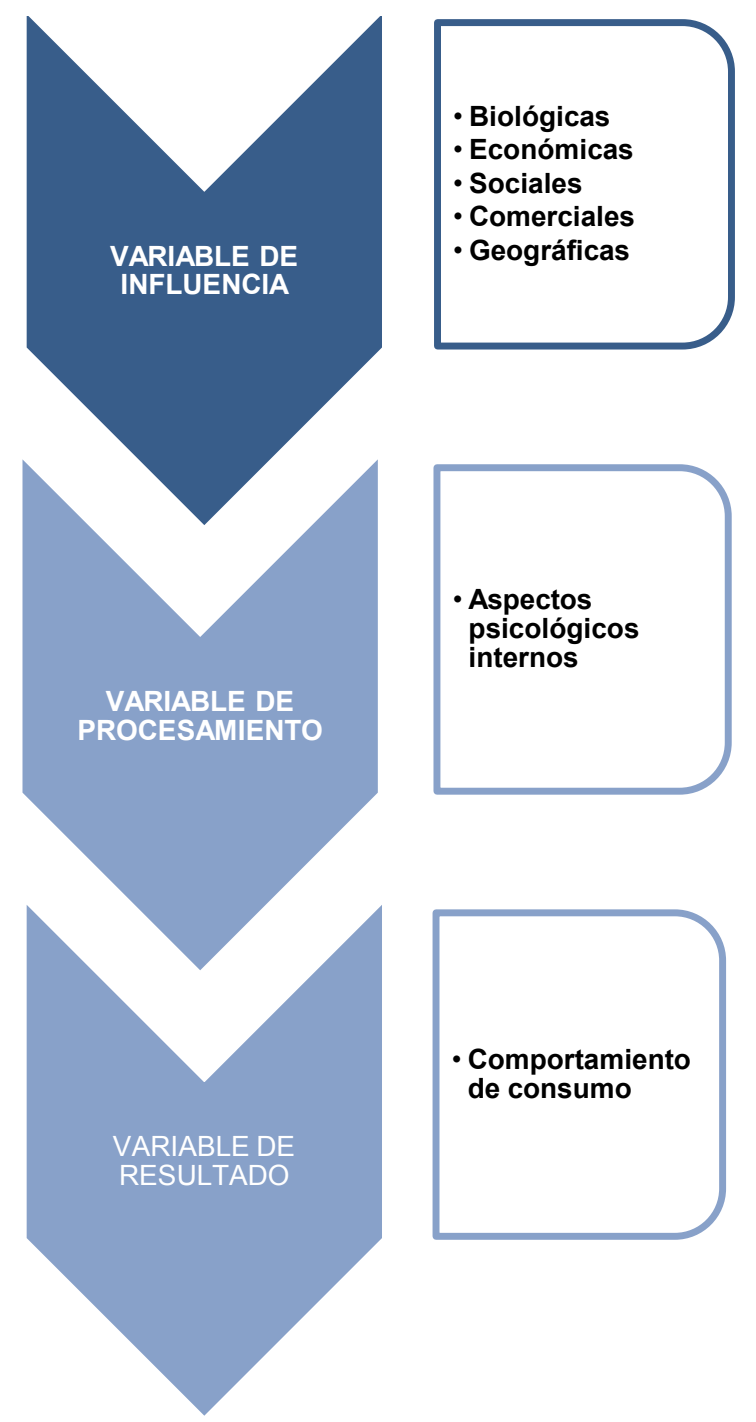

GRÁFICA 2 Variables del comportamiento (Cueva, 2011) 

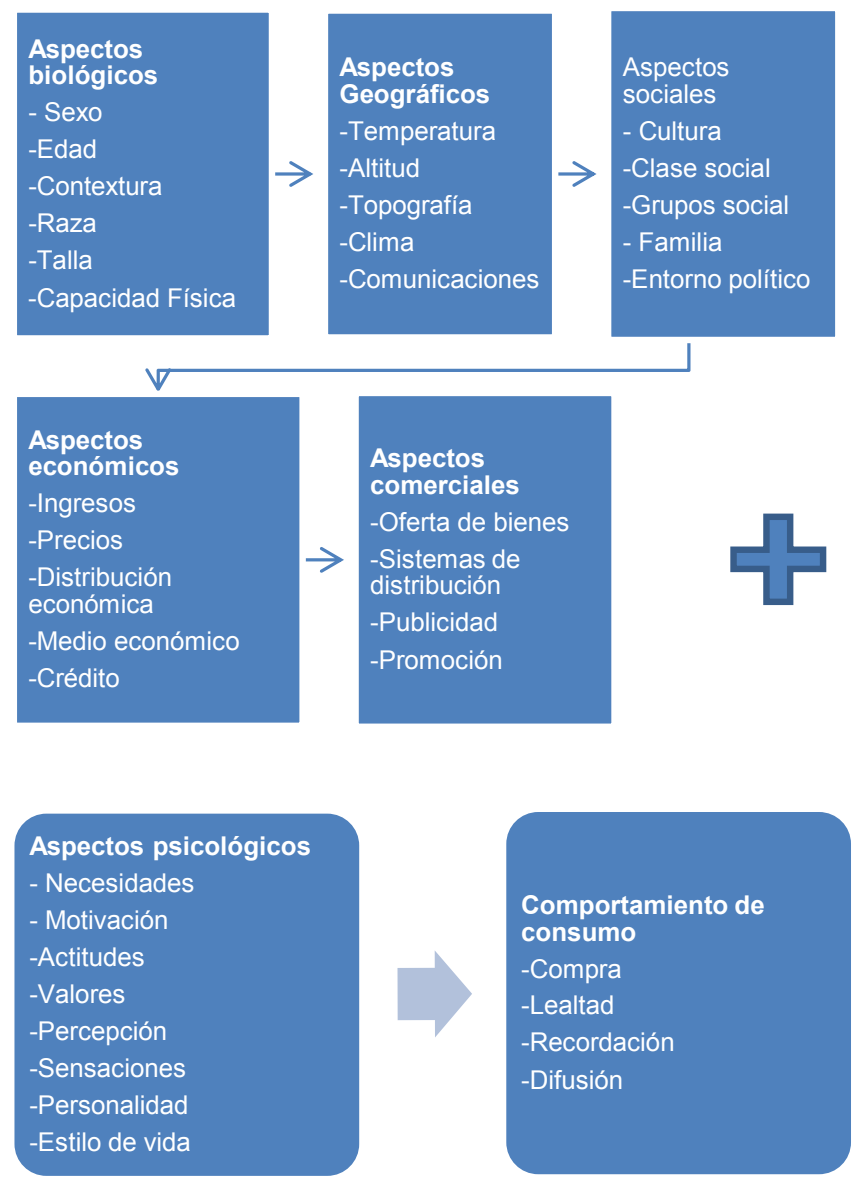

GRÁFICA 3 La influencia en el comportamiento del consumidor (Cueva, 2011)

\section{Emociones}

Las emociones humanas están básicamente clasificadas en tres categorías: motivación (sed, hambre, dolor, humor), básicas (feliz, triste, miedo, disgusto, ira, sorpresa) y sociales (encanto, pena, orgullo, culpa), de las cuales se puede establecer un conjunto de 6 emociones básicas que pueden ser generadas fácilmente (felicidad, tristeza, disgusto, miedo, alegría e ira) (AlMejrad, 2010). Otra forma de clasificar las emociones consiste en medir y contextualizar las emociones de acuerdo con diferentes dimensiones, cada una de las cuales representa un trato afectivo; por ejemplo, en el modelo excitación-emoción (Gráfica 4), la primera dimensión es la excitación-valencia, que explica el deseo, y la segunda explica la actividad fisiológica relacionada con el estado afectivo. La validez de esto radica en la relación entre la característica fisiológica y la excitación, debido a la activación del sistema nervioso automático cuando la emoción es generada, como se ilustra en la gráfica 4 (Bradley, 1994). 


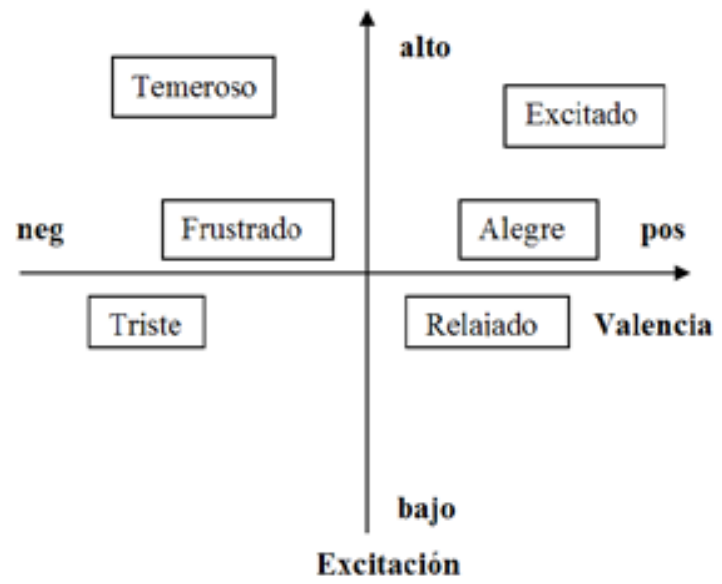

GRÁFICA 4 Modelo de Clasificación de emociones (Bradley, 1994)

\section{Círculo de Geneva}

En la gráfica 5 se muestra el círculo de Geneva el cual es un instrumento construido a partir de las teorías del color y de las emociones, pero empíricamente probado, a la hora de medir las reacciones emocionales con respecto a los objetos, acontecimientos y situaciones, ya que determinar una emoción y su duración es bastante complejo debido a que raramente se genera la misma respuesta en dos o más individuos. Este consiste en un círculo dividido en 20 ejes, correspondientes a las familias de emociones, y segmentado en 4 grupos que aúnan las emociones por opuestos así: i) Poder de alto control, ii) Valores negativos, iii) Poder de bajo control y iv) Valores positivos (Scherer, 2005).

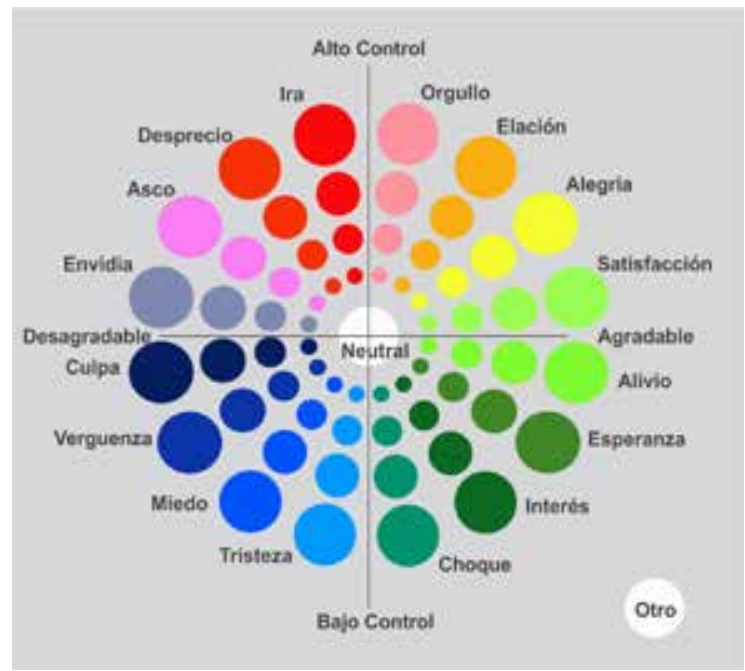

GRÁFICA 5. Círculo de Geneva (Scherer, 2005) 


\section{Metodología}

La metodología desarrollada en este estudio se describe en la gráfica 6 y para la cual se realizaron dos estudios: el primero basado en un análisis del color, emoción y moda a partir de datos obtenidos de encuestas, y el segundo mediante un análisis de color y emoción de información obtenida a partir de vídeos. A continuación, se detallan cada uno de estos análisis.

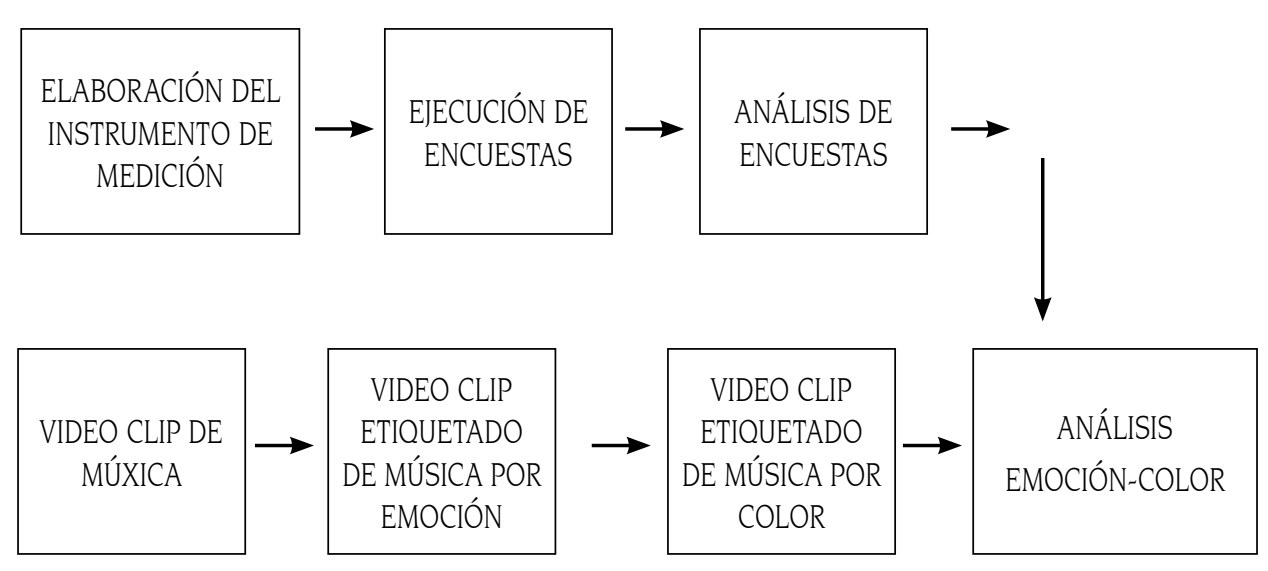

GRÁFICA 6 Metodología aplicada (elaboración propia)

Elaboración del instrumento de medición: se construyó un formulario, con preguntas que indagan acerca de las preferencias de color al momento de comprar, y su relación con algunas emociones como la alegría, el miedo, la excitación, la tristeza y la irritación.

Ejecución de encuestas: se aplicó el instrumento a una población o muestra no probabilística por conveniencia, de la Institución Universitaria Salazar y Herrera y del Instituto Tecnológico Metropolitano (ITM).

Análisis de encuestas: se tabularon las encuestas y se realizó un análisis comparativo y estadístico de la información obtenida.

Etiquetado vídeo clip música-color: se etiquetó la base de datos de vídeo clips musical de la base de datos DEAP, definido desde su escenario y puesta en escena por una tonalidad o varias en conjunto con el tema musical.

Etiquetado vídeo clip música-emoción: se etiquetaron los vídeo clips musicales de la base de datos DEAP, desde su escenario y puesta en escena por una emoción en conjunto con el tema musical.

Análisis emoción-color: se realizó un análisis comparativo en conjunto de las muestras musicales y su asociación emocional por color con los resultados de la encuesta teniendo como base el círculo cromático de Geneva, el cual permitió determinar el significado emocional a cada tonalidad del círculo; a continuación, se describe con mayor detalle. 


\section{Análisis del color, la emoción y la moda a partir encuestas}

La encuesta fue aplicada sobre una muestra que consta de un grupo de estudiantes inscritos en los programas de Diseño Gráfico, Diseño de Modas, Gestión Contable, Gestión Financiera, Administración de Empresas, Gestión Empresarial, Ingeniería Industrial, Ingeniería de Sistemas e Ingeniería Electrónica de la Institución Universitaria Salazar y Herrera (IUSH), y de los estudiantes de los programas Ingeniería Biomédica y Mantenimiento de Equipos Biomédicos, del Instituto Tecnológico Metropolitano (ITM) para un total de 293 estudiantes de la ciudad de Medellín y a la cual se le denomina como muestra 1 y se describe así: a) Elementos: Hombres y mujeres estudiantes entre 17 y 49 años de edad de la ciudad de Medellín. b) Unidad de muestra: 293 estudiantes de las instituciones universitarias IUSH e ITM. c) Extensión: Medellín (Colombia). Este tipo de muestreo no probabilístico por conveniencia no permite realizar inferencias sobre la población, pero permite caracterizar de manera efectiva la muestra a partir de los resultados obtenidos.

A partir de los datos obtenidos, se realizó un análisis de las encuestas de manera cuantitativa, para determinar cómo influye el color con el consumo de prendas de vestir, que en este caso hacen referencia a la moda y la emoción manifestada, para caracterizar al individuo en relación con su comportamiento de consumo.

\section{VIII.Análisis del color- emoción a partir de base de datos DEAP}

En esta parte del estudio, se utiliza la base de datos DEAP perteneciente a las universidades europeas: a) Queen Mary University de Londres, b) University of Twente de Holanda, c) University of Geneva y al d) EPFL de Suiza. Esta base de datos consta de registros de emociones obtenidos de 32 individuos (estudiantes de origen europeo), los cuales fueron sometidos a estímulos por medio de 40 vídeo clips y se encuentra ampliamente descrita en (Koelstra, y otros, 2012) y en el resto del documento se le denomina como muestra 2.

De los 40 vídeos de la muestra 2, se realizó una selección de 29 vídeos, los cuales fueron analizados, etiquetados y revisados por color y emoción, mediante la aplicación del circulo de Geneva, el cual desarrolla una categorización del color frente a la emoción.

\section{RESULTADOS}

Basados en la muestra 1 (encuesta), con respecto a la muestra 2 (vídeos) y el instrumento Geneva Emotion Wheel, se puede inferir que para la pregunta realizada en la encuesta: ¿Qué color le produce irritación? los datos arrojados, coincidieron con la emoción identificada en el gráfico Geneva, correspondiente al color naranja en un 15 \% relacionado emocionalmente con la irritación; además, se visualizó que para la pregunta ¿Qué color le causa alegría?, los datos arrojados, coincidieron con la emoción identificada en el gráfico Geneva, correspondiente al color amarillo en un porcentaje del 29 \% relacionado emocionalmente con la alegría.

En la tablal se muestran los resultados obtenidos basados en la muestra 1 (encuesta), con respecto a la muestra 2 (vídeos) y el instrumento Geneva; se puede inferir que para 
la pregunta realizada en la encuesta: ¿Qué color le produce tristeza? los datos arrojados coincidieron con la emoción identificada en el gráfico Geneva, correspondiente al color gris en un $26 \%$, relacionado emocionalmente con la tristeza; de igual manera, se puede inferir que los resultados en ambas muestras, con respecto a la emoción del miedo, tienen el color negro como predominante, identificado en la encuesta con un porcentaje de 45 \% ; también se visualizó que el color rojo corresponde a la emoción de la excitación, tanto en la muestra 2 (vídeos) como en la muestra 1 (encuesta), en esta última con un porcentaje del 55 \%; realizando otro análisis se puede inferir que la emoción de la alegría corresponde en ambas muestras al color amarillo que se destaca en la muestra 1 con un porcentaje del 29\% sobre los otros colores; algo similar ocurre con la emoción del odio: en ambas muestras está asociado con el color negro, teniendo como resultado en la muestra 2 (encuesta) un $35 \%$.

\begin{tabular}{|l|c|c|c|c|}
\hline \multicolumn{1}{|c|}{ PREGUNTA } & RESPUESTA & PORCENTAJE \% & BASE 1 & BASE 2 \\
\hline ¿Qué color le produce irritación? & Naranja & $15 \%$ & & $\mathrm{X}$ \\
\hline ¿Qué color le causa alegría? & Amarillo & $29 \%$ & $\mathrm{X}$ & $\mathrm{X}$ \\
\hline ¿Qué color le produce tristeza? & Gris & $26 \%$ & $\mathrm{X}$ & \\
\hline ¿Qué color le produce miedo? & Negro & $45 \%$ & $\mathrm{X}$ & \\
\hline ¿Qué color le produce excitación? & Rojo & $55 \%$ & $\mathrm{X}$ & \\
\hline ¿Qué color le produce odio? & Negro & $35 \%$ & $\mathrm{X}$ & \\
\hline
\end{tabular}

TABLA 1. Datos obtenidos del análisis la encuesta por emoción (elaboración propia)

En la tabla 2, se muestra, con base en la muestra 1 (encuesta), que frente a la pregunta de si el color influye en el ingreso a un establecimiento de consumo, la respuesta fue afirmativa con un $51 \%$, frente a la respuesta negativa de $48 \%$, lo que permite inferir que no es un comportamiento definitivo en la población ingresar o no a un determinado lugar solo por el color; sin embargo, frente a la pregunta, si el color influyó en la decisión de compra, la respuesta fue contundente, con $94 \%$ de respuestas afirmativas, y si han desistido de la compra por no encontrar el objeto en su color de preferencia, con un $65 \%$ de respuestas afirmativas, lo que permite observar que el color afecta las emociones y es decisivo a la hora de adquirir un objeto.

\begin{tabular}{|l|c|c|c|c|}
\hline \multicolumn{1}{|c|}{ PREGUNTA } & SÍ & PORCENTAJE \% & NO & PORCENTAJE \% \\
\hline $\begin{array}{l}\text { ¿Influyó el color en la decisión de ingreso al } \\
\text { establecimiento }\end{array}$ & 148 & $51 \%$ & 142 & $48 \%$ \\
\hline $\begin{array}{l}\text { ¿̇nfluyó el color en la decisión de compra de } \\
\text { un objeto? }\end{array}$ & 275 & $94 \%$ & 18 & $6 \%$ \\
\hline $\begin{array}{l}\text { ¿Ha desistido de comprar, por no encontrar } \\
\text { el color de su preferencia? }\end{array}$ & 190 & $65 \%$ & 103 & $35 \%$ \\
\hline
\end{tabular}

TABLA 2. Datos obtenidos del análisis la encuesta por actitud hacia el color (elaboración propia) 


\section{Conclusiones}

Se hizo un análisis del color, la emoción y la moda, basado en una encuesta regional sobre el color y la moda, y unos vídeos que relacionan las emociones. A partir de este análisis se descubre que existe una fuerte compatibilidad entre las emociones, el consumo de moda y el color, a pesar de los arraigos culturales y de los diferentes tipos de población analizados; es decir, el color muestra correspondencia en cuanto a su significado y está asociado a las emociones. Adicionalmente, a partir del análisis de las encuestas focalizadas en una única región, se demuestra una fuerte tendencia a obedecer las preferencias de color, en cuanto a la toma de decisiones de ingreso a establecimientos de consumo y al evento de la compra como tal, con resultados concluyentes y definitivos en su mayoría, lo que permite inferir que el consumo es afectado por el color y que se puede influenciar al consumidor, a tal punto de desistir de consumir un objeto por no encontrar su tonalidad favorita. Sin embargo, aunque se puede observar que hay coherencia y coincidencia con los significados en relación con las emociones proponentes del diagrama de Geneva (Geneva Emotion Wheel) y el análisis de la muestra 2 (videos), con respecto a la muestra 1 (encuesta), las cuales corresponden a regiones muy diversas no solo en edad, sino también en región, lo que es influyente, demuestra que el vínculo del color con el consumo de moda, no es consistente y genera conflictos con respecto a los significados del color, pero se concluye, que a raíz de los efectos del color en las emociones de los individuos, el objeto desde el diseño, debe contemplar la tendencias y la gamas cromáticas del color, con el fin de dar cobertura a la mayor cantidad de individuos. Como trabajo futuro, se propone realizar un análisis EEG-Emoción-Color-moda en tiempo real de muestras poblacionales más grandes, discriminadas por rangos de edad y sexo, que permita correlacionar estas variables desde un punto de vista menos cualitativo y contrastarlo respecto a los resultados obtenidos en este estudio y poder obtener una mayor generalidad en el estudio.

\section{Agradecimientos}

Este artículo, hace parte del proyecto de investigación "Análisis de la percepción del color sobre las emociones, por medio de técnicas inteligentes a partir de señales EEG y cómo esta se convierte en una variable del comportamiento de consumo de moda", que se realizó de manera interdisciplinaria entre miembros del grupo de investigación Código perteneciente a la Escuela de Artes, desde la línea el Diseño como medio de expresión sociocultural, y el Grupo GEA de la Escuela de Ingeniería desde la línea investigación de automática, ambos de la Institución Universitaria Salazar y Herrera. 


\section{REFERENCIAS}

AlMejrad, A. S. (2010). Human emotion detection using brainwave signals: A challenging. European Journal of Scientific Research, 640-659.

Álvarez Lara, O. (2011). Influencia del color en las preferencias de los consumidores. Revista Observatorio Calazanz Vol. II, N. ${ }^{\circ} 4,228-246$.

Babin, B. J., Hardesty, D., \& M., E. S. (2003). Color and shopping intentions: The intervening effect of price fairness and perceived affect. Journal of busines research, 56 (7), 54-551.

Bradley, M. M. (1994). Measuring emotion: The self-assessment manikin and the semantic differential. Journal of Behavior Therapy and Experimental Psychiatry, 25 (1), 49-59.

Cho, H.-S. E. (2005). Development of a macroscopic model on recent fashion trends on the basis of consumer emotion. . International Journal of Consumer Studies,29 (1), 17-33.

Cueva, R. A. (2011). Comportamiento del consumidor. México: Mc Graw Hill.

Desolneux, A., Moisan, L., E Morel, J.-M. (2008). From Gestalt Theory to Image Analysis A Probabilistic Approach. Springer.

Dr. Simon, M., E Goes, J. (2013). Dissertation and scholarly Research: Recipes for Success. Seatle: Copyrihted Material.

Gonzales Cuasante, J. M., \& Cuevas Riaño, M. d. (2005). Introducción al color. España: Ed. Akal S. A.

Koelstra, S., Mühl, C., Soleymani, M., Seok lee, J., Yazdani, A., Ebrahimi, T., . . Patras, L. (2012). A database for emotion analysis using physiological signals. IEEE Trans. Affective Computing 3, 1, 18-31.

Köhler, W. (1972). Psicología de la forma - Su tarea y sus últimas experiencias. Madrid: Biblioteca Nueva.

López Cruz, C. S. (2011). El papel del color en los espacios inmateriales: Caso en una interfaz histórica. Razón y Palabra 75, pp. 7-8.

Mangun, G. R. (2013). Cognitive Electrophysiology of attention. Academic Press.

Maya U. Shankar, C. A. (2009). The influence of color and label information on flavor perception. Chem Percept Springer, 53-58.

Morel, A. D.-M. (2004). Gestalt Theory and Computer Vision, in seeing, thinkins and knowing, Vol. 38. USA: Ed Springer Netherlands.

Morris, C. G. (2005). Introducción a la psicología. México: Prentice Hall.

Moszkowicz, J. (2011). Gestalt and Graphic Design: An explorations of the humanistic and Terapheutic effects of visual Organization. Design Issues, Vol. 27 N. ${ }^{\circ}$ 4, 56 -67.

Novaes, H. M. (1973). Psicología de la aptitud creadora. Buenos Aires: Kapelusz.

Oviedo, G. L. (2004). La definición del concepto de percepción en Psicología con base en la teoría Gestalt. Revista de estudios sociales N. ${ }^{\circ} 18,89-96$.

Puig Borrás, N. (2011). Emociones, arte y estética en la publicidad. Lecciones del portal (En Línea) Portal de comunicaciones InCom-UAB.

Roger Blackwell, J. F. (2009). Comportamaiento del consumidor. Florida: Cengage Learning Editores. 
Santalla de Banderalli, Z. (201 1). Efectos de la tipicidad del color y de la diagnosticidad del color sobre los juicios estéticos de agradabilidad de imágenes de objetos naturales y hechos por el hombre. Revista Anales Universidad Metropolitana Vol. 11 No 1, 31-51.

Satake, I. X. (2011). A comparative study of the emotional assessment of automotive exterior colors in Asia. Progress in Organic Coatings, 72 (3), 528-540.

Scherer, K. R. (2005). ¿Qué son las emociones? y ¿Cómo pueden ser medidos? SAGE Publications (Londres, Thousand Oaks, CA y Nueva Delhi, 695-729.

Shin, Y.-B., Woo, S.-H., Kim, D. H., Kim, J., Kim, J.-J., E Young Park, J. (2015). El efecto sobre las emociones y la actividad cerebral por la luz directa / indirecta en el entorno residencial. Neuroscience Letters Vol. 584 enero, 28-32.

Simón, C. A. (jul. 1990). In search of insight. Cognitive Psychology vol. 22 N. ${ }^{\circ} 3,374-1990$.

Yu, Y., Hui, C.-L., E Choi, T. -M. (2012). An empirical study of intelligent expert systems on forecasting of fashion color trend. Expert Systems with Applications, 39 (4), 4383 - 4389. 